\title{
Crystal Structure of 2-Aminobenzothiazolinium Perchlorate
}

\author{
Lesław SiERoń \\ Institute of General \& Ecological Chemistry, Technical University of Łódź, Żeromskiego 116, \\ 90-924 Łódź, Poland
} The crystal structure of the title compound, $\mathrm{C}_{7} \mathrm{H}_{7} \mathrm{~N}_{2} \mathrm{~S}^{+} \cdot \mathrm{ClO}_{4}^{-}$, contains cations and anions linked by $\mathrm{N}-\mathrm{H} \cdots \mathrm{O}$ hydrogen
bonds that form ribbons of alternating edge-fused $\mathrm{R}_{4}^{4}(12)$ and $\mathrm{R}_{4}^{4}(16)$ rings running along the $b$ axis.

(Received July 21, 2005; Accepted September 29, 2005; Published on web December 2, 2005)

\begin{abstract}
Aminothiazoles and their derivates belong to a group of biologically important compounds that have a very wide range of pharmacological activities, such as e.g. anti-inflammatory, immunoregulating, antiphlogistic-analgesics, antiulcer and psychotropic action. ${ }^{1}$ They have also found applications in the treatment of allergies, hypertension, inflammation and schizophrenia, as well as bacterial and HIV infections. ${ }^{2}$ This study was undertaken in order to understand the hydrogenbonding patterns in 2-aminobenzothiazolinium perchlorate (Fig. $1)$.
\end{abstract}

The title compound was prepared by reaction of the equimolar quantities of quinoline and perchloric acid (65\%). The resulting solid was recrystallized from water to afford crystals suitable for X-ray diffraction. The diffraction data were collected with KUMA KM4CCD diffractometer and CrysAlis CCD software. ${ }^{3}$ The structure was solved by a direct method using the SIR2002 program, ${ }^{4}$ and refined by a full-matrix least-squares method with anisotropic displacement parameters for the non-H atoms. All hydrogen atoms were located in a difference map, and their coordinates and isotropic displacement parameters were refined freely with resulting $\mathrm{C}-\mathrm{H}$ distances in the range of $0.88(4)$ $0.95(3) \AA$. The crystal data and the experimental details are summarized in Table 1. The final fractional atomic coordinates and the equivalent isotropic displacement parameter for nonhydrogen atoms are listed in Table 2. Selected bond distances and bond angles are listed in Table 3 , and the hydrogen-bonding geometric details are summarized in Table 4.

The presented title compound is composed of discrete 2aminobenzothiazolinium cations and perchlorate anions (Fig. 2). 2-Aminobenzothiazolinium and perchlorate ions are connected together by $\mathrm{N}-\mathrm{H} \cdots \mathrm{O}$ hydrogen bonds to form alternating edgefused $\mathrm{R}_{4}^{4}(12)$ and $\mathrm{R}_{4}^{4}(16)$ rings. The combination of these motifs produces one-dimensional ribbons of $\mathrm{C}_{2}{ }_{2}(8)$ graph-set lying approximately in the (103) plane and running along the $b$ axis, as shown in Fig. 3.

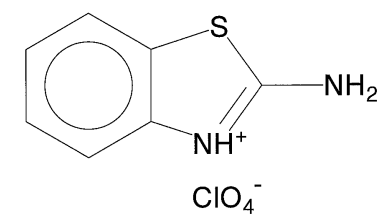

Fig. 1 Chemical structural diagram.
The 2-aminobenzothiazolium moiety is not ideally planar. Only the six $\mathrm{C}$ atoms of the benzene ring are exactly coplanar. The N2, S1, C1 and C6 atoms are displaced from the fivemembered ring plane by $-0.030(4),-0.009(1),-0.010(1)$, and $-0.009(1) \AA$, respectively.

The C1-S1 distance in the thiazole ring of $1.730(2) \AA$ is intermediate between those reported for a single $\mathrm{Csp}^{3}-\mathrm{S}$ (1.819 $\AA$ ) and a double $\mathrm{C}=\mathrm{S}$ bond $(1.61 \AA)$, but longer than the $\mathrm{C}-\mathrm{S}$ bond in thiophene (1.712 $\AA){ }^{7}$ The $\mathrm{O}$ atoms of the perchlorate anion are involved differently in the intermolecular hydrogen-

Table 1 Crystal and experimental data

\begin{tabular}{|c|c|}
\hline Empirical formula & $\mathrm{C}_{7} \mathrm{H}_{7} \mathrm{~N}_{2} \mathrm{~S}^{+} \cdot \mathrm{ClO}_{4}^{-}$ \\
\hline Formula weight & 250.67 \\
\hline Temperature & $296(2) \mathrm{K}$ \\
\hline Crystal size & $0.42 \times 0.28 \times 0.07 \mathrm{~mm}$ \\
\hline Crystal color & colorless \\
\hline Crystal description & prism \\
\hline Crystal system, space group, Z & triclinic, $P \overline{1}, 2$ \\
\hline$a=5.8021(3) \AA$ & $\alpha=85.805(4)^{\circ}$ \\
\hline$b=8.6623(4) \AA$ & $\beta=77.486(4)^{\circ}$ \\
\hline$c=10.2595(5) \AA$ & $\gamma=79.724(4)^{\circ}$ \\
\hline$V=494.99(4) \AA^{3}$ & \\
\hline Calculated density & $1.682 \mathrm{~g} / \mathrm{cm}^{3}$ \\
\hline 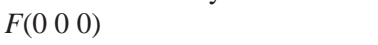 & 256 \\
\hline Radiation & $0.71073 \AA\left(\mathrm{Mo}-K_{\alpha}\right)$ \\
\hline$\mu\left(\mathrm{Mo}-K_{\alpha}\right)$ & $0.592 \mathrm{~mm}^{-1}$ \\
\hline Absorption correction & multi-scan \\
\hline Reflections collected / unique & $5188 / 1936\left[R_{\text {int }}=0.0138\right]$ \\
\hline $2 \theta_{\max }$ & $52.00^{\circ}$ \\
\hline Completeness to $\theta_{\max }$ & $99.8 \%$ \\
\hline$R$ indices $[I>2 \sigma(I)]$ & $R 1=0.0383, w R 2=0.1029$ \\
\hline$R$ indices (all data) & $R 1=0.0414, w R 2=0.1056$ \\
\hline Goodness-of-fit & 1.089 \\
\hline No. of reflections used & 1936 \\
\hline No. of parameters & 165 \\
\hline$(\Delta / \rho)_{\max }$ & $<0.001$ \\
\hline$(\Delta \rho)_{\max },(\Delta \rho)_{\min }$ & $0.49,-0.35 \mathrm{e}^{-3}$ \\
\hline Diffractometer & KUMA KM4CCD \\
\hline Monochromator & graphite \\
\hline Data collection program & CrysAlis CCD \\
\hline Structure determination: & SIR2002 \\
\hline Structure refinement: & SHELXL976 \\
\hline Structure drawing: & XSHELL and MERCURY \\
\hline CCDC Deposit No. & 284479 \\
\hline
\end{tabular}


Table 2 Atomic coordinates $\left(\times 10^{4}\right)$ and equivalent isotropic displacement parameters $\left(\AA^{2} \times 10^{3}\right)$

\begin{tabular}{ccccc}
\hline Atom & $x$ & $y$ & $z$ & $U_{\text {eq }}$ \\
\hline S1 & $2023(1)$ & $342(1)$ & $7583(1)$ & $45(1)$ \\
N1 & $1141(4)$ & $3244(2)$ & $8111(2)$ & $39(1)$ \\
N2 & $-1957(4)$ & $1883(3)$ & $9127(2)$ & $52(1)$ \\
C1 & $175(4)$ & $1953(3)$ & $8363(2)$ & $38(1)$ \\
C2 & $4778(5)$ & $4225(3)$ & $6829(3)$ & $48(1)$ \\
C3 & $6945(5)$ & $3790(4)$ & $5958(3)$ & $58(1)$ \\
C4 & $7706(5)$ & $2271(4)$ & $5532(3)$ & $61(1)$ \\
C5 & $6331(5)$ & $1109(4)$ & $5985(3)$ & $52(1)$ \\
C6 & $4160(4)$ & $1529(3)$ & $6854(2)$ & $39(1)$ \\
C7 & $3399(4)$ & $3065(3)$ & $7259(2)$ & $36(1)$ \\
Cl & $2352(1)$ & $2763(1)$ & $1475(1)$ & $41(1)$ \\
O1 & $2748(6)$ & $2508(3)$ & $2782(2)$ & $97(1)$ \\
O2 & $-110(4)$ & $3358(3)$ & $1494(4)$ & $101(1)$ \\
O3 & $3664(5)$ & $3914(3)$ & $786(2)$ & $85(1)$ \\
O4 & $3011(4)$ & $1345(2)$ & $749(2)$ & $64(1)$ \\
\hline
\end{tabular}

$U_{\mathrm{eq}}=(1 / 3) \sum_{i} \Sigma_{j} \sum_{i j} a_{i}^{*} a_{j}^{*} \boldsymbol{a}_{i} \cdot \boldsymbol{a}_{j}$

Table 3 Selected bond distances $(\AA)$ and angles $\left({ }^{\circ}\right)$

\begin{tabular}{llll}
\hline $\mathrm{S} 1-\mathrm{C} 1$ & $1.730(2)$ & $\mathrm{S} 1-\mathrm{C} 6$ & $1.759(2)$ \\
$\mathrm{N} 1-\mathrm{C} 1$ & $1.323(3)$ & $\mathrm{N} 1-\mathrm{C} 7$ & $1.398(3)$ \\
$\mathrm{N} 2-\mathrm{C} 1$ & $1.322(3)$ & $\mathrm{Cl}-\mathrm{O} 1$ & $1.404(2)$ \\
$\mathrm{Cl}-\mathrm{O} 2$ & $1.426(2)$ & $\mathrm{Cl}-\mathrm{O} 3$ & $1.417(2)$ \\
$\mathrm{Cl}-\mathrm{O} 4$ & $1.432(2)$ & & \\
& & & \\
$\mathrm{C} 1-\mathrm{S} 1-\mathrm{C} 6$ & $90.16(10)$ & $\mathrm{C} 1-\mathrm{N} 1-\mathrm{C} 7$ & $115.1(2)$ \\
$\mathrm{S} 1-\mathrm{C} 1-\mathrm{N} 1$ & $112.46(17)$ & $\mathrm{S} 1-\mathrm{C} 1-\mathrm{N} 2$ & $123.2(2)$ \\
$\mathrm{N} 1-\mathrm{C} 1-\mathrm{N} 2$ & $124.3(2)$ & $\mathrm{S} 1-\mathrm{C} 6-\mathrm{C} 5$ & $128.5(2)$ \\
$\mathrm{S} 1-\mathrm{C} 6-\mathrm{C} 7$ & $110.72(16)$ & $\mathrm{N} 1-\mathrm{C} 7-\mathrm{C} 2$ & $126.7(2)$ \\
$\mathrm{N} 1-\mathrm{C} 7-\mathrm{C} 6$ & $111.5(2)$ & & \\
\hline
\end{tabular}

Table 4 Hydrogen-bonding geometry $\left(\AA,^{\circ}\right)$

\begin{tabular}{ccccc}
\hline $\mathrm{D}-\mathrm{H} \cdots \mathrm{A}$ & $\mathrm{D}-\mathrm{H}$ & $\mathrm{H} \cdots \mathrm{A}$ & $\mathrm{D} \cdots \mathrm{A}$ & $\mathrm{D}-\mathrm{H} \cdots \mathrm{A}$ \\
$\mathrm{N} 1-\mathrm{H} 1 \cdots \mathrm{O} 2^{\mathrm{i}}$ & $0.72(3)$ & $2.28(3)$ & $2.937(3)$ & $153(3)$ \\
$\mathrm{N} 2-\mathrm{H} 6 \cdots 3^{\mathrm{ii}}$ & $0.83(4)$ & $2.33(4)$ & $3.069(4)$ & $148(3)$ \\
$\mathrm{N} 2-\mathrm{H} 7 \cdots 4^{i i \mathrm{ii}}$ & $0.88(3)$ & $2.09(3)$ & $2.955(3)$ & $166(3)$ \\
\hline
\end{tabular}

Symmetry codes: (i) $-x, 1-y, 1-z$; (ii) $x-1, y, 1+z$; (iii) $-x,-y, 1-z$

bond system. The $\mathrm{Cl}-\mathrm{O}$ bonds involved in hydrogen-bonding are distinctly longer $[1.417(2)-1.432(2) \AA]$ than the remaining not involved $\mathrm{Cl}-\mathrm{O} 1$ bond [1.404(2) $\mathrm{A}]$. A packing analysis does not indicate any significant stacking interactions or interactions with the $\mathrm{S} 1$ atom.

\section{Supplementary material}

Complete bond lengths and angles, coordinates and displacement parameters have been deposited at Cambridge Crystallography Data Centre (CCDC No. 284479).

\section{References}

1. Japan Patent, 1988, JP63060978.
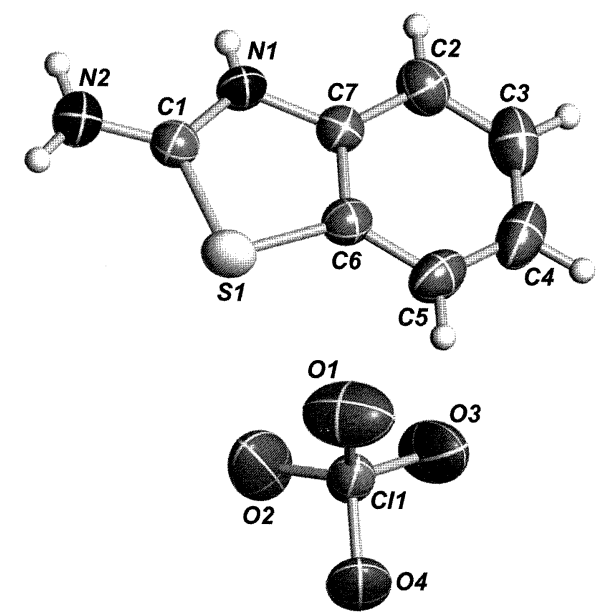

Fig. 2 View of the title compound with the atom numbering scheme. Displacement ellipsoids for non-hydrogen atoms are drawn at the $50 \%$ probability level.

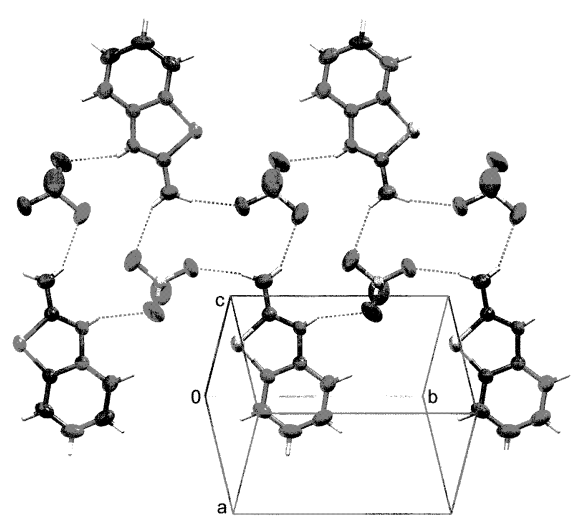

Fig. 3 Packing diagram showing a ribbon of hydrogen-bonded (dashed lines) cations and anions running along the $b$ axis.

2. P. C. Kearney, M. Fernandez, and J. A. Flygare, J. Org. Chem., 1998, 63, 196, and references therein.

3. Oxford Diffraction. CrysAlis CCD. Versions 1.171. Oxford Diffraction Ltd., Abingdon, Oxfordshire, England, 2004.

4. M. C. Burla, M. Camalli, B. Carrozzini, G. L. Casarano, C. Giacovazzo, G., Polidori, and R. Spagna, J. Appl. Cryst., 2003, 36, 1103.

5. M. C. Etter, J. C. MacDonald, and J. Bernstein, Acta Cryst. $B, \mathbf{1 9 9 0}, 46,256$.

6. G. M. Sheldrick, SHELXL97, A Program for Structure Determination, University of Göttingen, Germany, 1997.

7. F. H. Allen, O. Kennard, D. G. Watson, L. Brammer, A. G. Orpen, and R. Taylor, J. Chem. Soc. Perkin Trans. 2, 1987, $12, \mathrm{~S} 1$. 